\title{
Requerimiento de unidades calor en el cultivo del amaranto
}

Requirement of heat units in the crop of the amaranth

García-Pereyra J. ${ }^{1}$, Aviña-Martínez G. N. ${ }^{1}$, Rubio-Graciano R. B. ${ }^{1}$, Medina-Medrano R. ${ }^{1}$, Alvarado-Gómez $\mathrm{O}^{2}$, Alejandre-Iturbide $\mathrm{G}^{3}$

${ }^{1}$ Instituto Tecnológico de Valle del Guadiana de Durango. Km. 22.5., Carretera a México. Durango, Dgo. 34000. ${ }^{2}$ Facultad de Agronomía. Universidad Autónoma de Nuevo León, México. (omaralvarado@prodigy.net.mx ${ }^{3}$ Instituto Politécnico Nacional CIIDIR- IPN. Unidad Durango. (ghiturbide@hotmail.com)

${ }^{\square}$ Autor para correspondencia: jpereyra5@hotmail.com

Recibido: $19 / 01 / 2016$

Aceptado: 28/06/2016

\section{RESUMEN}

Se analizaron cinco genotipos de amaranto; cuatro de la especie Amaranthus hypochondriacus L., 153-5-3, 655, 653 y Criollo Tlaxcala y uno de la especie Amaranthus cruentus L., 33 en cuatro ambientes, contrastantes en clima y topografía ubicados en el norte de México. Los estudios se efectuaron entre el 15 de agosto de 2000 y el 15 de noviembre de 2002. El objetivo del presente trabajo fue: determinar la interacción genotipo por ambiente (A x G) en el requerimiento de unidades (UC) calor en el desarrollo fenológico del cultivo y el rendimiento de grano (RG). Se utilizó un diseño experimental de bloques completos al azar con tres repeticiones para diferentes ambientes. Los resultados indican que para MC (Madurez comercial), Criollo Tlaxcala de $A$. hypochondriacus fue el de mayor requerimiento con 977 UC en el ambiente A1, en A2; 655, 33, 653 presentaron comportamientos estadísticos similares con un requerimiento de 1475 UC cada uno, superando a Criollo Tlaxcala y 153-5-3 que requirieron de 1133 y 1473 UC, en A3 Criollo Tlaxcala fue el de menor requerimiento con 1384 UC, los demás genotipos estadísticamente fueron similares en el requerimiento de UC en promedio con 1568, para A4 los genotipos en estudio presentaron requerimientos en promedio de 315 UC. Los genotipos de mayor $R G(p \leq 0.05)$ fueron 153-5-3 de A. hypochondriacus en el ambiente A1 con $2144 \mathrm{~kg} \mathrm{ha}^{-1}$, en los ambientes A2 y A3 el genotipo 33 con 1637 y $1283 \mathrm{~kg} \mathrm{ha}^{-1}$, para A4 los genotipos 153-5-3 y 653 con y 1539 y $1562 \mathrm{~kg}$ $\mathrm{ha}^{-1}$ respectivamente.

Palabras clave: Amaranthus spp, unidades calor, emergencia de la planta, inicio de aparición de panícula, panícula completa, madurez comercial.

\begin{abstract}
Five amaranth genotypes were analyzed; four Amaranthus hypochondriacus L. species, 153-5-3, 655, 653, and Tlaxcala creole; the other species was Amaranthus cruentus L., thirty three in four environment, contrast climates and topography located in Northern Mexico. The studies were carried out between 15th August 2000 and 15th November 2002. The objective of this paper was: determine the interaction genotype by environment $(\mathrm{A} x \mathrm{G})$ on heat units required in the phenology
\end{abstract}


development from cultivates and the grain yielding of genotypes (RG). A complete random block experimental design with three repetitions for different environments was used. Results have shown that for MC (marketing ripeness) Tlaxcala Creole from A. hypochondriacus had a higher requirement with $977 \mathrm{UC}$ (heat units) in the A1 environment, in A2; 655, 33, 653 have shown similar statistical behaviors each one with a requirement of $1475 \mathrm{UC}$, overcoming to Tlaxcala Creole and to 153-5-3 which required 1133 and 1473 UC, for A3 Tlaxcala Creole had the least requirement with an average of $1384 \mathrm{UC}$, the rest of the genotypes were statistically similar of UC requirements with an average of 1568, for A4 each of the genotypes of the study shown average requirements of $315 \mathrm{UC}$. The higher RG ( $\mathrm{p}<0.05$ ) genotypes were 153-5-3 from A. hypochondriacus of environment A1 with $2144 \mathrm{~kg} \mathrm{ha}^{-1}$, for A2 and A3 environments genotype 33 with 1637 and $1283 \mathrm{~kg} \mathrm{ha}^{-1}$, for A4 the genotypes 153-5-3 y 653 with 1539 and $1562 \mathrm{~kg} \mathrm{ha}^{-1}$ respectively.

Keywords: Amaranthus spp., heat units, plant emergence, appearing of panicle, completed panicle, commercial ripeness.

\section{INTRODUCCIÓN}

Cada especie vegetal tiene temperaturas críticas que definen los requerimientos de calor necesarios para su crecimiento y desarrollo. Estas temperaturas, la mínima a la cual la planta crece, la óptima a la cual el crecimiento y desarrollo son más grandes y la máxima a la cual la planta crece (Ortiz, 1987). El concepto de unidades calor (UC) se desarrolló para estudiar la relación temperatura-planta, así como para medir las diferentes etapas del crecimiento cuando el cultivo se somete a diferentes ambientes (Baskerville y Emin, 1969). Las UC se basan en el crecimiento de la planta, que ocurre solamente cuando la temperatura diaria excede a la temperatura base del crecimiento de la planta; para el caso del amaranto los límites del desarrollo están entre 13.7 y 28.9 ${ }^{\mathrm{o}} \mathrm{C}$ y la temperatura media anual optima para el desarrollo del cultivo es de $21.3{ }^{\circ} \mathrm{C}$ (Reyna, 1986). El número de UC requeridas para un correcto desarrollo de la planta de amaranto depende de la especie empleada, del clima, la topografía y las localidades en las que se cultiva (Borodanenko et al., 1999). Ortega et al., (2003) mencionan que se requieren de 1629 unidades calor desde la siembra del grano hasta la madurez comercial para los genotipos de Amaranthus hypochondriacus L., pudiéndose obtener un rendimiento de grano en promedio 346 a $360 \mathrm{~g} \mathrm{~m}^{-2}$, a una densidad de población de
100,000 plantas $\mathrm{ha}^{-1}$, estos resultados se obtuvieron en el ambiente de Texcoco, región situada en los Valles Centrales del Estado de México en el año de 2001. Matteucci (1998), que realizó una evaluación de tres especies de amaranto en la pampa Argentina, reporta que en los genotipos de Amaranthus cruentus L., la formación de PC fue a los 45 días después de la siembra a un fotoperiodo corto menor a 13 horas, la madurez comercial fue hasta los 118 días para obtener un RG de 2000 a 3600 $\mathrm{kg} \mathrm{ha}^{-1}$. El objetivo del presente trabajo fue:

(1) determinar la interacción genotipo por ambiente $(\mathrm{A} \times \mathrm{G})$ en el requerimiento de unidades calor en cuatro estados del desarrollo fenológico del cultivo de cinco variedades de amaranto: siembra-emergencia de la planta (E), siembra-inicio de aparición de panícula (IAP), siembra-panícula completa (PC), siembra-madurez comercial (MC), sembrados en cuatro ambientes contrastantes en clima, suelo y altitud en el norte de México: (2) determinar el rendimiento de grano (RG) para cada ambiente de prueba. La hipótesis general es que presentan menores requerimientos de unidades calor y un mayor rendimiento de grano los genotipos de A. cruentus y A. hypochondriacus durante los ciclos de siembra de primavera-verano que los sembrados en los ciclos de siembra de otoño-invierno. 


\section{MATERIALES Y MÉTODOS}

Se utilizaron cinco genotipos de amaranto, cuatro de la especie $A$. hypochondriacus que se denominan colectas 153-5-3, 655, 653 y Criollo Tlaxcala y un genotipo denominado colecta 33 perteneciente a la especie A. cruentus. Los genotipos se sembraron durante los ciclos de primavera-verano (PV) del año 2000, ambiente 1 (A1); otoño-invierno (OI) en el año 2001, ambiente 2 (A2); otoño-invierno (OI) en el año 2002, ambiente 3 (A3), todos ellos sembrados en la localidad de Marín, N. L. Una última siembra fue en la localidad del Valle del Guadiana, Durango, durante el ciclo de primavera-verano (PV) del año 2002, ambiente 4 (A4), no se empleó ningún tratamiento como testigo. Se utilizó un diseño experimental de bloques completos al azar para diferentes localidades, con tres repeticiones (Olivares, 1994). El tamaño de la parcela fue de $6 \mathrm{~m} \mathrm{x} 4 \mathrm{~m}\left(24 \mathrm{~m}^{2}\right)$. Las actividades de campo para los experimentos realizados en la localidad de Marín, N. L., fueron similares en los tres ambientes de prueba y consistieron en rastra, desterronado, surcado y bordeado, sembrado, y cuatro riegos espaciados. En la localidad del Valle del Guadiana, Dgo., la siembra se condujo bajo condiciones de secano y no se aplicó fertilizante en ninguna etapa del cultivo ni ningún tratamiento a la semilla. Para cada ciclo las fechas de siembra y de cosecha fueron: (1) primavera-verano (PV) año 2000: siembra 04/08 y cosecha $04 / 12$; (2) otoñoinvierno (OI) año 2001: siembra 17/03 y cosecha 17/08; (3) otoño-invierno (OI) año 2002: siembra $21 / 03$ y cosecha $27 / 08$; (4) primavera-verano (PV) año 2002: siembra

19/06 y cosecha 17/11 En los cuatro ambientes de estudio para el combate de las plagas se utilizó $250 \mathrm{ml} \mathrm{ha}^{-1}$ del insecticida Metasystox $^{\circledR}$ en la etapa de IAP del cultivo. Se calcularon las unidades calor mediante el procedimiento descrito por (Baskerville and Emin, 1969). La fórmula aplicada para el cálculo: requerimiento de unidades calor (UC) = AT-BT en donde AT: es el promedio de la temperatura diaria en la estación de desarrollo del cultivo $\left(\mathrm{T}_{\text {Máxima }}+\mathrm{T}_{\text {Mínima }}\right) / 2$;
BT: Temperatura base (temperatura bajo la cual el crecimiento no ocurre) en las diferentes etapas fenológicas del cultivo de amaranto: siembra- emergencia de la planta (E), siembra-inicio de aparición de panícula (IAP), siembra-panícula completa (PC) y siembra-madurez comercial (MC). El rendimiento de grano ( $\mathrm{RG})$ se determinó cosechando a mano las plantas individuales solo de los surcos centrales de cada parcela para un total de 24 plantas espaciadas $30 \mathrm{~cm}$ cada una de ellas con una superficie de evaluación de $10 \mathrm{~m}^{2}$. La semilla se separó mediante una máquina trilladora para granos. Se efectuó el análisis de varianza a una $\mathrm{p} \leq$ 0.05 para el requerimiento de unidades calor para los genotipos en los ambientes de prueba mediante el software de diseños experimentales (1994), y cuando se detecto diferencia estadística significativa, en la interacción genotipos $\mathrm{x}$ ambientes $(\mathrm{G} \times \mathrm{A}$ ) se procedió a efectuar la comparación de medias por DMS (diferencia mínima significativa) mediante la prueba protegida de Steel y Torrie (1981). Para evaluar el rendimiento de grano (RG) se efectuó el análisis de varianza para cada ambiente experimental en forma separada y la significancia estadística a una $\mathrm{P} \leq 0.05$ mediante la comparación de medias por DMS.

\section{RESULTADOS}

\section{Condiciones ambientales}

En el ciclo de siembra del ambiente (A1) se presentaron 29 días con temperaturas superiores a $34^{\mathrm{O}} \mathrm{C}$ y 18 días con menores a $14^{\mathrm{O}} \mathrm{C}$; siendo el mes de agosto el más cálido, con temperaturas hasta de $43^{\circ} \mathrm{C}$. En este ambiente la siembra se efectuó en la segunda semana del mes de agosto. El fotoperiodo durante el ciclo del cultivo en promedio fue de 11.4 h efectuándose la cosecha en el mes de diciembre. La precipitación total para este ciclo agrícola fue de $280 \mathrm{~mm}$ y la evaporación de $600 \mathrm{~mm}$. Para los ambientes A2 y A3, la temperatura máxima fue $38^{\circ} \mathrm{C}$ y la mínima $15^{\circ} \mathrm{C}$, siendo el mes de mayo el más cálido. En estos ambientes la siembra se efectuó en la tercera semana de marzo, a un fotoperiodo de 12.9 h en promedio, la cosecha se efectuó 
García-Pereyra et al., 2016

en la segunda semana de julio, cuando el fotoperiodo era de $13 \mathrm{~h}$. La precipitación total en A2 fue $111 \mathrm{~mm}$ y la evaporación 916 mm. Para A3 la precipitación total fue 128 $\mathrm{mm}$ y la evaporación $1143 \mathrm{~mm}$. En A4 la siembra se realizó en el mes de junio a un fotoperiodo de $13.3 \mathrm{~h}$ y a una temperatura de $30^{\circ} \mathrm{C}$ la cosecha se efectuó en el mes de noviembre cuando el fotoperiodo era $11.3 \mathrm{~h}$ y la temperatura promedio en este mes fue de $12.5^{\circ} \mathrm{C}$, la precipitación acumulada en este ciclo de cultivo fue $497.6 \mathrm{~mm}$ y la evaporación 1034 mm (Cuadro 1).

Cuadro 1. Condiciones ambientales durante el crecimiento de amaranto sembrado en el norte de México, años 2000, 2001 y 2002.

\begin{tabular}{|c|c|c|c|c|c|c|c|c|}
\hline \multirow[t]{2}{*}{ Ambiente } & \multirow[t]{2}{*}{ Mes } & \multicolumn{2}{|c|}{$\begin{array}{r}\text { Temperatura } \\
\left({ }^{\circ} \mathrm{C}\right)\end{array}$} & \multirow[t]{2}{*}{$\begin{array}{l}\text { Promedio } \\
\left({ }^{\circ} \mathrm{C}\right)\end{array}$} & \multirow[t]{2}{*}{$\begin{array}{c}\text { Evaporación } \\
\text { total } \\
(\mathrm{mm})\end{array}$} & \multirow[t]{2}{*}{$\begin{array}{c}\text { Evaporación } \\
\text { media } \\
(\mathrm{mm})\end{array}$} & \multirow[t]{2}{*}{$\begin{array}{c}\text { Precipitación } \\
\text { total } \\
(\mathrm{mm})\end{array}$} & \multirow[t]{2}{*}{$\begin{array}{l}\text { Horas Luz } \\
\text { promedio } \\
\text { del mes }\end{array}$} \\
\hline & & Max. & Mín. & & & & & \\
\hline \multicolumn{9}{|l|}{ PV 2000} \\
\hline & Ago. & 43.0 & 18.6 & 30.8 & 236.6 & 7.6 & 75.0 & 12.8 \\
\hline & Sept. & 31.0 & 19.9 & 25.5 & 106.3 & 3.5 & 124.0 & 12.0 \\
\hline & Oct. & 29.3 & 16.5 & 22.9 & 122.4 & 3.9 & 12.0 & 11.3 \\
\hline & Nov. & 25.4 & 12.5 & 18.9 & 68.2 & 2.2 & 64.0 & 10.6 \\
\hline & Dic. & 18.7 & 6.9 & 12.8 & 67.4 & 2.1 & 5.0 & 10.3 \\
\hline \multicolumn{9}{|l|}{ OI 2001} \\
\hline & Mar. & 24.2 & 11.3 & 17.8 & 102.4 & 3.3 & 31.0 & 12.0 \\
\hline & Abr. & 30.1 & 18.0 & 24.1 & 171.7 & 5.7 & 17 & 12.5 \\
\hline & May. & 37.8 & 20.0 & 29.2 & 213.6 & 6.8 & 29 & 13.3 \\
\hline & Jun. & 34.8 & 22.5 & 29.1 & 209.5 & 6.9 & 21 & 13.4 \\
\hline & Jul. & 38.1 & 22.3 & 30.2 & 219.5 & 7.0 & 13 & 13.3 \\
\hline \multicolumn{9}{|l|}{ OI 2002} \\
\hline & Mar. & 29.8 & 12.4 & 21.1 & 196.4 & 6.3 & 3.0 & 12.4 \\
\hline & Abr. & 27.8 & 20.3 & 27.8 & 248.0 & 7.7 & 5.0 & 12.5 \\
\hline & May. & 38.1 & 21.4 & 29.3 & 279.8 & 9.0 & 26.0 & 13.2 \\
\hline & Jun. & 37.4 & 23.1 & 30.2 & 264.2 & 8.8 & 94.0 & 13.3 \\
\hline & Jul. & 35.0 & 22.2 & 28.4 & 154.6 & 4.9 & 198 & 13.3 \\
\hline \multicolumn{9}{|l|}{ PV } \\
\hline \multirow[t]{7}{*}{2002} & Jun. & 30 & 13 & 21.5 & NR & NR & NR & 13.3 \\
\hline & Jul. & 30.0 & 8.0 & 19.0 & 245.0 & 8.1 & 54.1 & 13.6 \\
\hline & Ago. & 30.0 & 7.0 & 18.5 & 186.8 & 6.2 & 103.9 & 13.2 \\
\hline & Sept. & 27.0 & 7.0 & 17.0 & 176.9 & 5.8 & 140.3 & 12.7 \\
\hline & Oct. & 25.0 & 2.0 & 13.5 & 138.4 & 4.6 & 94.5 & 11.7 \\
\hline & Nov. & 25.0 & 0.0 & 12.5 & 151.9 & 5.0 & 50.0 & 11.3 \\
\hline & Dic. & 22.0 & 4.5 & 13.2 & 135.4 & 4.5 & 54.8 & 10.7 \\
\hline
\end{tabular}

Fuente: Departamento de Meteorología y Climatología de la Facultad de Agronomía de la Universidad Autónoma de Nuevo León. 2000, 2001 y 2002., Comisión Nacional del Agua, Durango. Departamento de Aguas Superficiales e Ingeniería de ríos 2002. 
García-Pereyra et al., 2016

\section{Análisis de varianza para unidades calor}

Existió una significancia estadística $(\mathrm{p} \leq 0.5)$ para la interacción A X G en unidades calor en todas las etapas de desarrollo del cultivo, ya que los genotipos presentaron diferencias numéricas para UC debido a lo contrastante de los climas y la topografía, en la localidad de Marín, N. L., la altitud es de $900 \mathrm{msnm}$ y las temperaturas son mas secas y calientes en la etapa de desarrollo del cultivo y para la localidad del Valle del Guadiana la altitud es de $1850 \mathrm{msnm}$ y las temperaturas son mas frescas (Cuadro 2).

Cuadro 2. Cuadrados medios para UC en cuatro etapas del desarrollo en genotipos de amaranto, evaluados en el norte de México.

\begin{tabular}{|c|c|c|c|c|c|}
\hline Fuente de variación & GL & $\bar{E}$ & IAP & $\overline{\mathrm{PC}}$ & $\mathrm{MC}$ \\
\hline Repeticiones & 2 & & & & \\
\hline Ambientes(A) & 3 & $92.90^{\mathrm{NS}}$ & $436932.0 *$ & $2581725.25^{*}$ & $4551376 *$ \\
\hline Error (a) & 8 & 0.78 & 9.50 & 6.00 & 8.0 \\
\hline Genotipos (G) & 4 & $32.16^{\mathrm{NS}}$ & $117306.50^{\mathrm{NS}}$ & $26350.00^{\mathrm{NS}}$ & $41957.00^{\mathrm{NS}}$ \\
\hline A X G & 12 & $240.52 *$ & $66521.5^{*}$ & $94583.0 *$ & $16166.33^{*}$ \\
\hline Error (b) & 32 & 1.15 & 7.12 & 4.50 & 7.25 \\
\hline Total & 59 & & & & \\
\hline $\mathrm{CV}(\%)$ & & 2.01 & 0.46 & 0.24 & 0.255 \\
\hline
\end{tabular}

$\mathrm{GL}=$ grados de libertad; $\mathrm{E}=$ Emergencia; $\mathrm{IAP}=$ inicio de aparición de panícula; $\mathrm{PC}=$ panícula completa; $\mathrm{MC}=$ madurez comercial; ${ }^{*}=$ significativo $(\mathrm{p} \leq 0.05) ;{ }^{\mathrm{NS}}=$ no significativo $(\mathrm{p}>0.05)$

\section{Siembra-emergencia de la planta $(E)$}

Por existir significancia estadística $(\mathrm{p} \leq 0.5)$ para la interacción A X G en todas las etapas de desarrollo del cultivo, se realizó la comparación de medias (Cuadro 3), Para esta variable los mayores requerimientos de unidades calor fueron para el genotipo 33 de A. cruentus en A1 y A2 con 70 y 71 UC respectivamente. Para A3 el genotipo 655 de A. hypochondriacus fue el de mayor requerimiento de unidades calor con $56.6 \mathrm{y}$ para A4 los genotipos de A. hypochondriacus evaluados tuvieron similares requerimientos de unidades calor con 54. La diferencia numérica observada en UC para el genotipo 33 en la misma localidad de Marín N. L., en el ambiente A2 (71 UC) y A3 (48 UC) se explica a que en $\mathrm{A} 2$ la temperatura promedio del mes de marzo fue de $17.8^{\circ} \mathrm{C}$ y en $\mathrm{A} 3$ de $21.1^{\circ} \mathrm{C}$, en este ambiente A3 la evaporación diaria y la precipitación acumulada fueron mayores que en A2 con diferencias numéricas de precipitación de $28 \mathrm{~mm}$ y evaporaciones de $3 \mathrm{~mm}$. 
Cuadro 3. Requerimiento de UC para E en cinco genotipos de amaranto evaluados en cuatro ambientes del norte de México.

\begin{tabular}{lcccc}
\hline Genotipos & A1 & A2 & A3 & A4 \\
\hline 33 & $70.0 \mathrm{a}^{*}$ & $71.0 \mathrm{a}$ & $48.0 \mathrm{f}$ & $45.0 \mathrm{~g}$ \\
655 & $57.0 \mathrm{~b}$ & $55.0 \mathrm{c}$ & $56.6 \mathrm{~b}$ & $54.0 \mathrm{c}$ \\
653 & $57.3 \mathrm{~b}$ & $57.0 \mathrm{~b}$ & $50.0 \mathrm{e}$ & $54.0 \mathrm{c}$ \\
Criollo Tlaxcala & $52.0 \mathrm{~d}$ & $52.0 \mathrm{~d}$ & $50.0 \mathrm{e}$ & $54.0 \mathrm{c}$ \\
$153-5-3$ & $51.3 \mathrm{~d}$ & $52.0 \mathrm{~d}$ & $50.0 \mathrm{e}$ & $54.0 \mathrm{c}$ \\
DMS $(0.05)$ & 1.76 & & &
\end{tabular}

A1= ambiente primavera-verano (PV) año 2000 Marín, N. L., A2 y A3 = ambiente otoño-invierno (OI) años 2001 y 2002 Marín, N. L., A4 = ambiente primavera-verano (PV) año 2002, Valle del Guadiana, Durango. * Promedios con diferente letra en una columna son estadísticamente diferentes (DMS, $\mathrm{p} \leq 0.05$ ).

\section{Siembra-inicio de aparición de panícula (IAP)}

Para IAP, el genotipo Criollo Tlaxcala de A. hypochondriacus requirió 642.3 unidades calor para la emergencia de la panícula en A1, en A2, los genotipos 33, 655 y 153-5-3 requirieron $853.6,851.3$ y $852.3 \mathrm{UC}$ respectivamente. Para A3 los genotipos 653, 33 y 655 requirieron de 732.6 UC cada uno, para esta variable de siembra-inicio de aparición de panícula. En A4 los genotipos 653,33 y 655 estadísticamente fueron similares en requerimientos de $\mathrm{UC}$, estos genotipos presentaron menores requerimientos de unidades calor en la localidad del Valle del Guadiana, Durango que en la localidad de Marín N. L., (Cuadro 4), esto se explica a que en la localidad de Marín N. L., las temperaturas promedio para el desarrollo del cultivo son mas altas que las reportadas en la localidad del Valle del Guadiana, Durango en el ciclo de desarrollo del cultivo, también se debe a que en el caso del genotipo 33 está adaptado a condiciones mas secas y calientes como las que se presentaron en el ambiente de PV 2000 en la localidad de Marín N. L., los genotipos 653,33 y 655 presentaron requerimientos de 232 unidades calor en promedio para la variable de siembra-inicio de aparición de panícula en A4.

Cuadro 4. Requerimiento de UC para IAP en cinco genotipos de amaranto probados en cuatro ambientes del norte de México.

\begin{tabular}{lllll}
\hline Genotipos & A1 & A2 & A3 & A4 \\
\hline Criollo Tlaxcala & $642.3 \mathrm{c}^{*}$ & $492.3 \mathrm{~g}$ & $553.0 \mathrm{f}$ & $217.0 \mathrm{i}$
\end{tabular}




$\begin{array}{llrrr}653 & 632.0 \mathrm{~d} & 493.3 \mathrm{~g} & 732.6 \mathrm{~b} & 232.0 \mathrm{~h} \\ 33 & 604.0 \mathrm{e} & 853.6 \mathrm{a} & 732.6 \mathrm{~b} & 233.4 \mathrm{~h} \\ 655 & 604.0 \mathrm{e} & 851.3 \mathrm{a} & 732.6 \mathrm{~b} & 232.0 \mathrm{~h} \\ 153-5-3 & 604.0 \mathrm{e} & 852.3 \mathrm{a} & 485.0 \mathrm{c} & 210.3 \mathrm{j}\end{array}$

$\operatorname{DMS}(0.05) \quad 4.43$

A1= ambiente primavera-verano (PV) año 2000 Marín, N. L., A2 y A3 = ambiente otoño-invierno (OI) años 2001 y 2002 Marín, N. L., A4 = ambiente primavera-verano (PV) año 2002, Valle del Guadiana, Durango. * Promedios con diferente letra en una columna son estadísticamente diferentes $(\mathrm{DMS}, \mathrm{p} \leq 0.05)$.

\section{Siembra-panícula completa (PC)}

Para PC se presentan los requerimientos de UC para los cinco genotipos en los cuatro ambientes de estudio (Cuadro 5). En A1, 655 de A. hypochondriacus presentó los mayores requerimientos de unidades calor con 639.3 seguido del genotipo 33 de A. cruentus con 922, estadísticamente los genotipos 153-5-3, Criollo Tlaxcala y 653 presentaron comportamientos similares con 912 y 910 UC respectivamente. En A2 los genotipos 153-53 , Criollo Tlaxcala y 653 , estadísticamente fueron similares con requerimientos de 1270, 1270 y 1273 UC respectivamente, en este ambiente 655 y 33 requirieron $603 \mathrm{UC}$ cada uno para esta variable de PC. En A3 los genotipos de A. hypochondriacus 153-5-3, Criollo Tlaxcala y 653 estadísticamente fueron similares con un requerimiento de 1435 UC en promedio cada uno para PC. Para A4; 153-5-3, Criollo Tlaxcala y 653 fueron los de mayor requerimiento de unidades calor 316 UC en promedio, en este ambiente 655 y 33 fueron los de menor requerimiento de unidades calor para llegar a panícula completa. En A4, todos los genotipos de prueba requirieron menos unidades calor para llegar a PC; esto se debe a que su formación se dio entre los 77 y 84 días después de la siembra a inicios de septiembre con una temperatura máxima de $28.5^{\circ} \mathrm{C}$ y un fotoperiodo de $12.7 \mathrm{~h}$.

Cuadro 5. Requerimiento de UC para PC en genotipos de amaranto

probados en el norte de México.

\begin{tabular}{lllll}
\hline Genotipos & A1 & A2 & A3 & A4 \\
\hline 655 & $939.3 \mathrm{~d}^{*}$ & $603.0 \mathrm{~g}$ & $1041.6 \mathrm{c}$ & $295.0 \mathrm{j}$ \\
33 & $922.0 \mathrm{e}$ & $603.6 \mathrm{~g}$ & $1152.0 \mathrm{~b}$ & $312.0 \mathrm{i}$ \\
$153-5-3$ & $912.0 \mathrm{f}$ & $1270.0 \mathrm{~b}$ & $1436.0 \mathrm{a}$ & $316.0 \mathrm{~h}$ \\
Criollo Tlaxcala & $910.0 \mathrm{f}$ & $1270.0 \mathrm{~b}$ & $1435.0 \mathrm{a}$ & $316.6 \mathrm{~h}$ \\
653 & $910.0 \mathrm{f}$ & $1273.3 \mathrm{~b}$ & $1435.3 \mathrm{a}$ & $314.0 \mathrm{~h}$
\end{tabular}


A1= ambiente primavera-verano (PV) año 2000 Marín, N. L., A2 y A3 = ambiente otoño-invierno (OI) años 2001 y 2002 Marín, N. L., A4 = ambiente primavera-verano (PV) año 2002, Valle del Guadiana, Durango. * Promedios con diferente letra en una columna son estadísticamente diferentes (DMS, $\mathrm{p} \leq 0.05)$.

\section{Siembra-madurez comercial (MC)}

Los mayores requerimientos de UC para MC, para los cinco genotipos de estudio, se presentaron en los ambientes A2 y A3 con temperaturas de cosecha en promedio de 30 ${ }^{\circ} \mathrm{C}$ y con un fotoperiodo de $13.3 \mathrm{~h}$; para $\mathrm{A} 4 \mathrm{el}$ requerimiento de UC para $\mathrm{MC}$ fue un $75 \%$ menor con respecto a $\mathrm{A} 1, \mathrm{~A} 2$ y $\mathrm{A} 3$ debido principalmente a que la temperatura de cosecha fue de $13.2{ }^{\circ} \mathrm{C}$ y el fotoperiodo en $11.3 \mathrm{~h}$ (Cuadro 6).

Cuadro 6. Requerimiento promedio de UC para la variable MC en cinco genotipos de amaranto probados en cuatro ambientes del norte de México.

\begin{tabular}{lcccc}
\hline Genotipos & A1 & A2 & A3 & A4 \\
\hline 655 & $960.5 \mathrm{f}^{*}$ & $1475.3 \mathrm{~b}$ & $1568.0 \mathrm{a}$ & $316.6 \mathrm{~g}$ \\
33 & $959.5 \mathrm{f}$ & $1475.5 \mathrm{~b}$ & $1568.6 \mathrm{a}$ & $319.0 \mathrm{~g}$ \\
$153-5-3$ & $959.0 \mathrm{f}$ & $1473.6 \mathrm{~b}$ & $1567.0 \mathrm{a}$ & $316.0 \mathrm{~g}$ \\
Criollo Tlaxcala & $977.3 \mathrm{e}$ & $1133.6 \mathrm{~d}$ & $1384.0 \mathrm{c}$ & $316.0 \mathrm{~g}$ \\
653 & $960.0 \mathrm{f}$ & $1475.0 \mathrm{~b}$ & $1564.6 \mathrm{a}$ & $315.0 \mathrm{~g}$ \\
DMS (0.05) & 4.4 & & &
\end{tabular}

A1= ambiente primavera-verano (PV) año 2000 Marín, N. L., A2 y A3 = ambiente otoño-invierno (OI) años 2001 y 2002 Marín, N. L., A4 = ambiente primavera-verano (PV) año 2002, Valle del Guadiana, Durango. * Promedios con diferente letra en una columna son estadísticamente diferentes (DMS, $\mathrm{p} \leq 0.05$ ).

\section{Duración de las distintas etapas de crecimiento del cultivo}

Para (A1) el genotipo 33 de A. cruentus en $\mathrm{MC}$ requirió de 110 días, los genotipos de A. hypochondriacus, en este mismo ambiente requirieron de 107 días (Figura 1). 


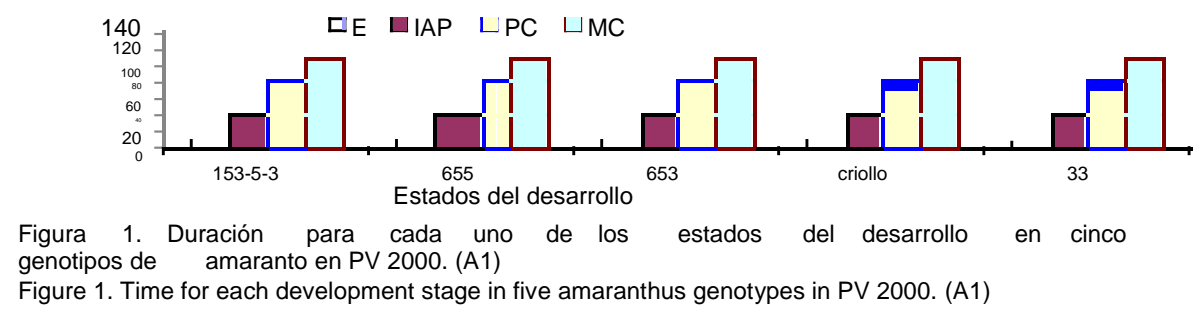

\section{Rendimiento de grano}

Para el ambiente A1 el genotipo 153-5-3 de A. hypochondriacus con $2144 \mathrm{~kg} \mathrm{ha}^{-1}$ fue el de mayor rendimiento de grano, en A2 y A3 el genotipo 33 de A. cruentus con 1637 y

Cuadro 7. RG en genotipos de amaranto sembrados en cuatro ambientes del norte de México.
$1283 \mathrm{~kg} \mathrm{ha}^{-1}$ y en A4 se presentó un RG de y 1539 y $1562 \mathrm{~kg} \mathrm{ha}^{-1}$ en los genotipos de A. hypochondriacus $153-5-3$ y 653 (Cuadro 7).

\section{Ambientes}

$\begin{array}{lllll}\text { Genotipos } & \text { A1 } & \text { A2 } & \text { A3 } & \text { A4 }\end{array}$

$$
\mathrm{RG}\left(\mathrm{kg} \mathrm{ha}^{-1}\right)
$$

\begin{tabular}{lcccc}
\hline $153-5-3$ & $2144 \mathrm{a}^{*}$ & $179 \mathrm{~b}$ & $345 \mathrm{~b}$ & $1539 \mathrm{a}$ \\
653 & $1968 \mathrm{a}$ & $318 \mathrm{~b}$ & $253 \mathrm{~b}$ & $1562 \mathrm{a}$ \\
Criollo Tlaxcala & $1788 \mathrm{~b}$ & $181 \mathrm{c}$ & $993 \mathrm{a}$ & $856 \mathrm{~b}$ \\
655 & $1443 \mathrm{c}$ & $306 \mathrm{~b}$ & $104 \mathrm{c}$ & $814 \mathrm{~b}$ \\
33 & $1015 \mathrm{~d}$ & $1637 \mathrm{a}$ & $1283 \mathrm{a}$ & $790 \mathrm{~b}$ \\
DMS (0.05) & 407 & 208 & 903 & 712
\end{tabular}

A1= ambiente primavera-verano (PV) año 2000 Marín, N. L., A2 y A3 = ambiente otoño-invierno (OI) años 2001 y 2002 Marín, N. L., A4 = ambiente primavera-verano (PV) año 2002, Valle del Guadiana, Durango. * Promedios con diferente letra en una columna son estadísticamente diferentes (DMS, $\mathrm{p} \leq 0.05$ )

\section{DISCUSIÓN}

En siembra-panícula completa resultados similares son reportados por Boradonenko et al., (1999) en donde mencionan requerimientos de fotoperiodo de 11 horas y temperaturas frescas para PC en los genotipos de A. hypochondriacus pero un mayor 
requerimiento de UC y de fotoperiodo para los genotipos de la especie A. cruentus. (Reyna, 1986), establece una necesidad de un fotoperiodo mayor para una buena madurez comercial en los genotipos de $A$. hypochondriacus cuando se siembran en zonas templadas y en menor cantidad para los genotipos de A. cruentus cuando crecen en zonas secas y cálidas. En siembra-madurez comercial (MC) en cuanto a UC para los cuatro ambientes de estudio difieren de los reportados por (Ortega et al. 2003), ellos mencionan que el requerimiento fue de 1629 UC para MC en genotipos de $A$. hypochondriacus en siembras realizadas en Texcoco, Estado de México. Matteucci (1998), evaluó tres diferentes genotipos de $A$. cruentus y tres de $A$. hypochondriacus en la pampa de Argentina y encontró que para $A$. cruentus, su MC fue de 118 días y para $A$. hypochondriacus de 128 días. Resultados similares para MC, son los reportados por Peña (1996), en una evaluación de los mismos genotipos bajo el ambiente de Tlaxcala, región ubicada en los Valles Centrales de

México, donde encontró en $A$. hypochondriacus 149 días para MC y 112 días para los genotipos de A. cruentus. (García et al., 2004) en trabajos realizados con los mismos genotipos de este estudio en la localidad de Marín N. L., región situada en el norte de México, encontraron que los genotipos de A. hypochondriacus L., fueron altamente sensibles en la etapa de floración, a las altas temperaturas y al fotoperiodo largo, incidente que ocasionó androesterilidad y bajos rendimientos de grano. (García et al., 2004) encontraron rendimientos de grano

\section{CONCLUSIONES}

Este estudio reviste importancia ya que se determina la utilidad en cuanto a la siembra de los genotipos de A. hypochondriacus en climas templados con inicio de temperaturas de siembra altas y con cosecha en temperaturas bajas como ocurre en la localidad del Valle del Guadiana de Durango. Los genotipos evaluados presentaron las mayores demandas de UC para MC del grano, durante los ciclos de siembra de Otoño-
(RG) de $1637 \mathrm{~kg} / \mathrm{ha}$ en siembras de otoño invierno efectuadas en la localidad de Marín, N. L. con el genotipo 33 de A. cruentus, debido a que la etapa de floración del amaranto fue superior a $40^{\circ} \mathrm{C}$, los genotipos sembrados de $A$. hypochondriacus variedades 653 y 655 presentaron RG de 306 y $312 \mathrm{~kg} / \mathrm{ha}$ en el mismo ambiente de prueba. (Barrales, 1997) establece que el amaranto es una planta de fotoperiodo corto y que días con nubosidad presentan condiciones desfavorables en el rendimiento de grano, ya que se favorece el crecimiento secundario, la producción en los genotipos de A. cruentus y A. hypochondriacus oscila entre los $250 \mathrm{y}$

$2400 \mathrm{~kg} \mathrm{ha}{ }^{-1}$ conclusiones de trabajos desarrollados con evaluación de 10 genotipos en la localidad de Chapingo, México. (Monsalvo y Oliver, 2004) en siembras efectuadas la primer semana del mes de junio en el estado de México con un genotipo de A. hypochondriacus encontraron rendimientos de grano de $1354 \mathrm{~kg} \mathrm{ha}^{-1}$, pero con tendencias a obtener menores rendimientos de grano a medida que el fotoperiodo se alarga a mas de 11 horas. Torres et al., (2006) encontraron rendimientos de grano de $4000 \mathrm{~kg} / \mathrm{ha}$ en genotipos de A. hypochondriacus, y de 1200 $\mathrm{kg} / \mathrm{ha}$ para $A$. cruentus a DP de 375000 plantas/ha en siembras realizadas en Tulyehualco D.F. (García et al., 2009) encontraron que en el Valle del Guadiana, Durango, región del norte centro de México, el mejor rendimiento de grano fue para $A$. cruentus (en fechas de siembra del 15 de marzo al 15 de abril) cuando la estación de crecimiento del cultivo se inicio con temperaturas bajas y en ascenso.

Invierno 2001 y 2002 en la localidad de Marín N. L. Los genotipos de $A$. hypochondriacus ensayados requirieron de menores UC para MC del grano cuando son sembrados en los ciclos de siembra de primavera-verano 2000 y 2002, en la localidad del Valle del Guadiana, Durango. El mayor rendimiento de grano fue para los genotipos de A. hypochondriacus 153-5-3 y 653 para los dos ambientes de prueba de la localidad del Valle del Guadiana, Durango, en donde presentan su mayor expresión genética 
debido a la adaptación de la especie a climas templados. Los genotipos de A. cruentus no se recomiendan para su siembra cuando el objetivo es el rendimiento de grano en ambientes con presencia de clima templado, tal y como se presenta en el Valle del Guadiana, Durango.

\section{LITERATURA CITADA}

Alejandre Iturbe, Gabriel y Gómez Lorence, Federico. 1999. Cultivo del amaranto en México. Universidad Autónoma Chapingo. Colección Cuadernos Universitarios. Serie Agronomía No. 12. Chapingo, Estado de México.

Baskerville, L.; Emin, P. 1969. Rapid estimation of heat unit accumulation from maximum and minimum temperatures. Ecol. 50: 514-517. https://doi.org/10.2307/1

Barrales, J. 1997. Agronomía Mesoamericana $8(2)$ :

127-130.

https://doi.org/10.15517/am.v8i2.24

Borodanenko, A.; González C.; y Ramírez, R. 1999. Avances del programa de mejoramiento genético de amaranto en la Universidad de Guanajuato. In: El Amaranto Cultivo promisorio del siglo XXI. pág.: 83-88.

García, J.; Valdes, C.; Olivares, E.; Alvarado, O.; Medrano, I.; Alejandre, G. 2004. Evaluación de genotipos de amaranto para adaptabilidad productiva en el noreste de México. Rev. Fitotec. Méx. Vol. 27 (Núm. Especial 1):53-56, 2004.

García, J.; Valdes, C.; Olivares, E,; Alvarado O,; Alejandre G, Salazar E,; Medrano H. 2009. Rendimiento de grano y calidad del forraje de

amaranto (Amaranthus spp.) cultivado a diferentes densidades en el noreste de México. Phyton vol. 78. Pág. 53-60.

https://doi.org/10.32604/phyton.200

Matteucci, S. 1998. Potencial productivo del amaranto en la pampa ondulada Argentina: Comportamiento de seis germoplasmas. Facultad de Agronomía. (LUZ).15: 560 - 570.
García-Pereyra et al., 2016

\section{AGRADECIMIENTOS}

Gabriel Alejandre Iturbide agradece al Instituto Politécnico Nacional por los apoyos económicos otorgados y a la COFAA por la beca de exclusividad.

Monsalvo, C.; Oliver, G. 2004. Producción de amaranto (Amaranthus hypochondriacus L.) a tres fechas de siembra en Huazulco, Temoac, Morelos. UAEMEX. Memorias de redes ambientales

Ortega, D.; Escalante, A.; Santos, A. 2003.

Rendimiento, eficiencia agronómica del nitrógeno $\mathrm{y}$ eficiencia en el uso del agua en amaranto en función del manejo del cultivo. Terra Latinoamericana 22: 109-116.

Olivares, E. 1994. Software de Diseños Experimentales FAUANL versión 2.5.Facultad de Agronomía. U. A. N. L. Marín, N. L. 215 p.

Ortiz S., C. A. 1987. Elementos de agrometeorología cuantitativa. Tercera edición. Departamento de Suelos. Universidad Autónoma Chapingo. Chapingo, México. 326 p.

Peña, R. 1996. Comportamiento productivo agronómico de cuatro genotipos de amaranto (Amaranthus spp) en seis fechas de siembra. Memorias VII Congreso Nacional de Investigación y Desarrollo Tecnológico Agropecuario. Roque, Celaya, Gto. 156 p.

Reyna, T. 1986. Requerimientos climáticos para el cultivo del amaranto (Amaranthus spp) en México. Primer Seminario Nacional del Amaranto. Chapingo, México. pp. 81-89.

Steel, R.; Torrie J. 1981. Principles and procedures of statistics, a biometrical approach. McGraw Hill Co., $625 \mathrm{p}$.

Torres, S.G., S.A. Trinidad, T.R. Trujillo, H.C. Juárez, A.E. Estrada y F.L. 


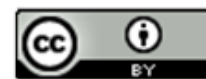

Este tex to está protegido por una licencia licencia Creative Commons 4.0

Usted es libre para Compartir —copiar y redistribuir el material en cualquier medio o form ato- y Adaptar el documento —remezclar, transformar y crear a partir del material- para cualquier propósito, , incluso para fines comerciales, siempre que cumpla la condición de:

Atribución: Usted debe dar crédito a la obra original de manera adecuada, proporcionar un enlace a la licencia, e indicar si se han realizado cambios. Puede hacerlo en cualquier form a razonable, pero no de form a tal que sugiera que tiene el apoyo del licenciante o 10 recibe por el uso que hace de la obra.

Resumencielicencia - Textocompletodelalicencia 